\title{
Long-term influence of fluid inertia on the diffusion of a Brownian particle
}

\author{
Giuseppe Pesce, ${ }^{1, *}$ Giorgio Volpe, ${ }^{2}$ Giovanni Volpe, ${ }^{3,4}$ and Antonio Sasso ${ }^{1}$ \\ ${ }^{1}$ Dipartimento di Fisica Università degli studi di Napoli, Complesso Universitario Monte S. Angelo, Via Cintia 80126, Napoli, Italy \\ ${ }^{2}$ Department of Chemistry, University College London, 20 Gordon Street, London WC1H OAJ, United Kingdom \\ ${ }^{3}$ Soft Matter Lab, Department of Physics, Bilkent University, Ankara 06800, Turkey \\ ${ }^{4}$ UNAM-National Nanotechnology Research Center, Bilkent University, Ankara 06800, Turkey
}

(Received 27 February 2014; published 20 October 2014)

\begin{abstract}
We experimentally measure the effects of fluid inertia on the diffusion of a Brownian particle at very long time scales. In previous experiments, the use of standard optical tweezers introduced a cutoff in the free diffusion of the particle, which limited the measurement of these effects to times comparable with the relaxation time of the fluid inertia, i.e., a few milliseconds. Here, by using blinking optical tweezers, we detect these inertial effects on time scales several orders longer up to a few seconds. The measured mean square displacement of a freely diffusing Brownian particle in a liquid shows a deviation from the Einstein-Smoluchowsky theory that diverges with time. These results are consistent with a generalized theory that takes into account not only the particle inertia but also the inertia of the surrounding fluid.
\end{abstract}

DOI: 10.1103/PhysRevE.90.042309

PACS number(s): 82.70.-y, 05.40.-a, 07.10.Pz, 47.15.G-

\section{INTRODUCTION}

Often a single parameter is key to the description of the motion of a Brownian particle: the particle's diffusion coefficient $D$. Its correct estimation assumes therefore a pivotal importance in many soft matter systems that are characterized thanks to the observation of the motion of a microscopic probe, such as the measurement of nanoscopic forces [1-3] and of the thermodynamic properties of microscopic systems [4-7].

In the original picture conceived by Albert Einstein, microscopic particles undergo a never-ending random motion due to collisions with the molecules of the fluid where they are immersed [8]. This picture is formalized by the original Langevin equation $[9,10]$ :

$$
\underbrace{m \ddot{x}(t)}_{\text {inertia }}=\underbrace{-\gamma \dot{x}(t)}_{\text {friction }}+\underbrace{\gamma \sqrt{2 D} W(t)}_{\text {diffusion }},
$$

where $x(t)$ is the particle's position; $m, \gamma$, and $D$ are, respectively, its mass, friction coefficient, and diffusion coefficient; and $W(t)$ is a Gaussian white noise [11,12]. The motion of a microscopic particle is, therefore, governed by two counteracting forces: the friction between the particle and the surrounding viscous fluid modeled by the Stokes drag and the random thermal force modeled by the white noise. Einstein's relation

$$
D=\frac{k_{\mathrm{B}} T}{\gamma},
$$

connects these two forces to the particle's average kinetic energy per degree of freedom, i.e., $k_{\mathrm{B}} T / 2$, where $k_{\mathrm{B}}$ is the Boltzmann constant and $T$ is the absolute temperature. The relation in Eq. (2) shows that the particle's kinetic energy is limited by the dissipation associated to its collisions with the surrounding fluid molecules [8].

When dealing with particles in the low Reynolds number regime [13], the inertial term in Eq. (1) is often neglected. This introduces an error in the estimation of $D$, which

\footnotetext{
*giuseppe.pesce@ fisica.unina.it
}

nevertheless, decreases exponentially in $\tau$ with a characteristic time dictated by the momentum relaxation time $\tau_{\mathrm{m}}=m / \gamma$. Thanks to this exponential decay, considering that $\tau_{\mathrm{m}}$ is in the order of microseconds for small colloidal particles and typical experiments are performed on time scales of milliseconds and longer, this error can be safely ignored in most experiments, and the motion of the particle is often considered overdamped [14]. The particle's motion, however, is also influenced by the surrounding fluid that has to move in order to refill the space left free by the particle's displacement [15-19]. This second inertial effect, known as hydrodynamic memory, develops over the time scale of the fluid momentum relaxation time $\tau_{\mathrm{f}}=R^{2} \rho_{\mathrm{f}} / \eta$, where $\rho_{\mathrm{f}}$ is the density of the fluid and $\eta$ its viscosity [19]. The effect of the fluid inertia only decays polynomially with $\tau^{-1 / 2}$ and, therefore, has a long-term influence on the determination of $D$.

In previous experiments, the use of standard optical tweezers limited the measurement of these inertial effects at very short time scales, i.e., $t \approx \tau_{\mathrm{m}}, \tau_{\mathrm{f}}$, because of the cutoff in the particles free diffusion introduced by the optical trap [20-24], while the polynomial weak decrease of the hydrodynamic memory means that these effects can have influences at time scales that are comparable to those of standard experiments, i.e., up to several seconds. Here, by measuring the diffusive motion of a Brownian particle over several seconds with a blinking optical tweezers setup [25,26], we experimentally demonstrate that the polynomial weak decay of the correction to the diffusion coefficient due to the presence of hydrodynamic memory produces measurable effects on time scales several orders of magnitude longer than those previously reported, up to a few seconds.

\section{THEORY}

In order to formalize the above discussion, we consider the mean square displacement (MSD) of a particle. The MSD quantifies how far a particle moves from its initial position and is an experimentally measurable quantity closely related to the particle's diffusion coefficient $D$. In one dimension, the MSD can be calculated as the time average of the particle's position 
$\left\langle x^{2}\right\rangle(\tau)=\overline{x(t+\tau) x(t)}[7,12]$, where the overbar represents time average, and $D$ can be estimated as

$$
D=\lim _{\tau \rightarrow+\infty} \frac{\left\langle x^{2}\right\rangle(\tau)}{2 \tau} \approx \frac{\left\langle x^{2}\right\rangle(\tau)}{2 \tau},
$$

where $\tau$ is large. In practice, $\tau$ in Eq. (3) cannot be taken to infinity, as it is limited by the experimentally accessible data range. For a massless particle $(m=0)$, whose motion obeys Eq. (1), the estimation of the diffusion coefficient using Eq. (3) is always exact at any $\tau$ and is not affected by experimental limitations in the acquisition of long data series. For a particle with inertia $(m \neq 0)$, this estimation leads to an exponentially decreasing error in $\tau$. Nonetheless, this error can be safely neglected in most experiments as it decays on a time scale given by the momentum relaxation time $\tau_{\mathrm{m}}$, which, as we have seen, is on the order of microseconds.

However, the MSD for a particle whose motion obeys the Langevin equation corrected to take into account the hydrodynamic memory effect $[15,16,19]$ is

$$
\left\langle x^{2}\right\rangle(\tau)=2 D \tau\left\{1-\sqrt{\frac{4 \tau_{\mathrm{f}}}{\pi \tau}}-\frac{8 \tau_{\mathrm{f}}}{9 \tau}+\frac{\tau_{\mathrm{m}}}{\tau}+\epsilon(\tau)\right\},
$$
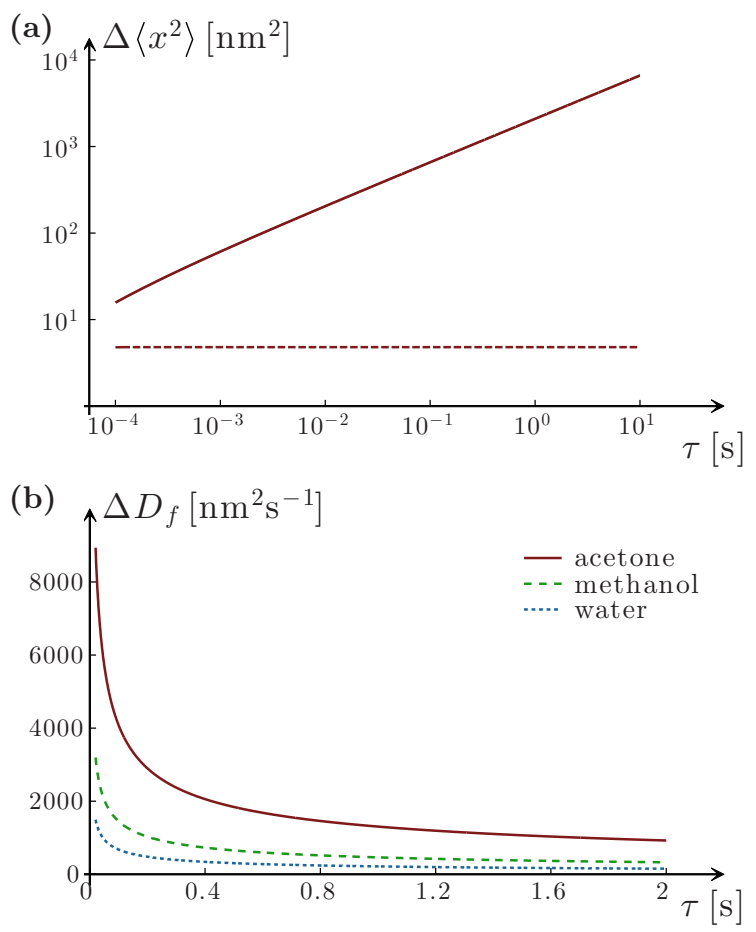

FIG. 1. (Color online) (a) Error in the estimation of the $\Delta\left\langle x^{2}\right\rangle(\tau)$ in the case of a melamine particle $(d=8.1 \mu \mathrm{m})$ taking into account its mass (dashed line) and taking into account also the fluid inertia (solid line). While the former quickly reaches a plateau, the latter diverges as a function of $\tau$. (b) Error in the estimation of the diffusion coefficient $D$ for the same particle immersed in the various fluids used in this work taking into account the presence of the fluid inertia [Eq. (5)], which decays polynomially with $\tau^{-1 / 2}$. When only the particle inertia is taken into account, the error on the estimation of $D$ decays exponentially with characteristic time $\tau_{\mathrm{m}}$ so that the error is negligible on the plotted scales.

where $\epsilon(\tau)$ is a correction term relevant only for very short times, i.e., for $\tau \ll \tau_{\mathrm{f}}, \tau_{\mathrm{m}}$, and thus can be safely neglected in the following discussion. In this case, as shown by the solid line in Fig. 1(a), the error on the MSD diverges as a function of $\tau$. The error in the estimation of $D$ can be derived using Eq. (3) and is

$$
\Delta D_{f}(\tau)=D-D_{f}(\tau)=D\left(\sqrt{\frac{4 \tau_{\mathrm{f}}}{\pi \tau}}+\frac{8 \tau_{\mathrm{f}}}{9 \tau}-\frac{\tau_{\mathrm{m}}}{\tau}\right),
$$

which decays polynomially with $\tau^{-1 / 2}$. Therefore, this effect can have an influence at time scales comparable to standard experiments, as shown by the solid lines in Fig. 1(b) for the different fluids used in the experiments shown in this article.

\section{EXPERIMENT}

The experimental setup that we used in order to measure these inertial effects at long time scales is schematically shown in Fig. 2(a). It consists of an optical tweezer built on a highstability homemade inverted optical microscope equipped with a high-numerical-aperture water-immersion objective lens (Olympus, UPLAPO60XW3, NA = 1.20). The optical trap was generated by a frequency and amplitude stabilized

(a)
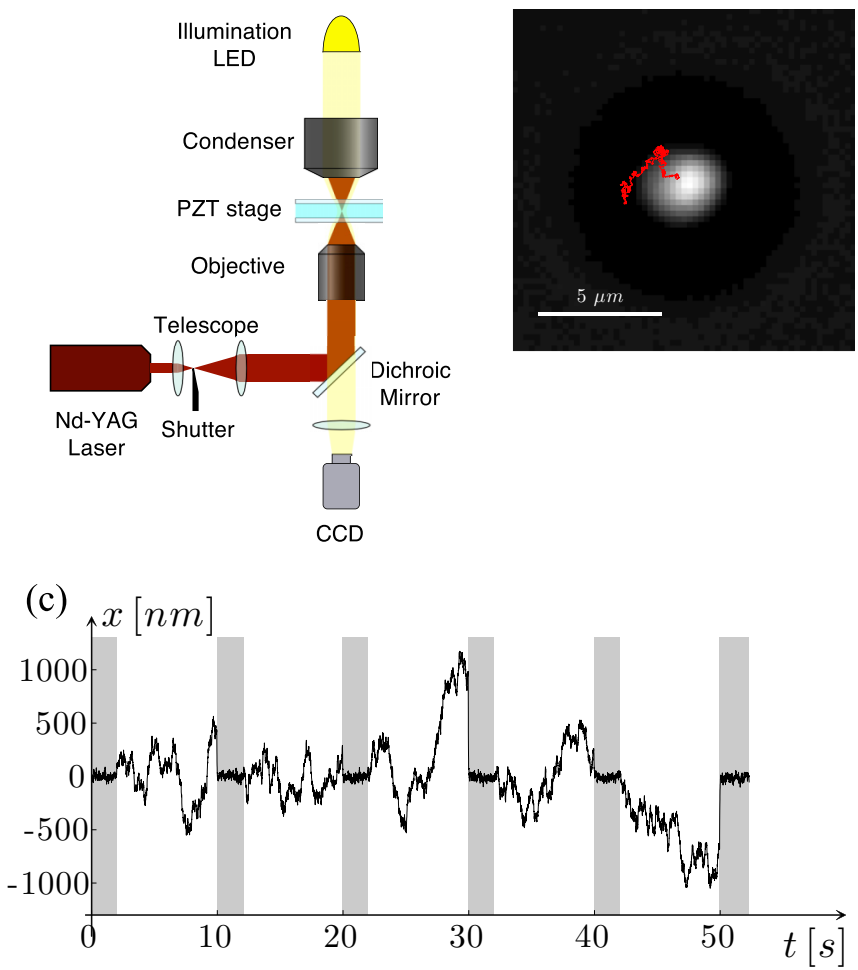

FIG. 2. (Color online) (a) The setup consists of a blinking optical tweezer combined with a fast digital video acquisition system. (b) Image and trajectory of a particle freely diffusing after being released from the trap. (c) Example of the $x$ component of the trajectory acquired while the particle is trapped and released repeatedly by the optical tweezers. The gray-shaded areas represent the periods of time when the trapping laser is on. 
TABLE I. Properties of the particles used in the experiments. Errors on diameters are reported by the manufacturers.

\begin{tabular}{lcc}
\hline \hline Material & $d(\mu \mathrm{m})$ & $\rho\left(\mathrm{g} / \mathrm{cm}^{3}\right)$ \\
\hline Polystyrene & $5.0 \pm 0.1$ & 1.05 \\
Melamine & $8.1 \pm 0.1$ & 1.51 \\
Licristar & $3.10 \pm 0.05$ & 1.41 \\
Silica & $4.8 \pm 0.2$ & 1.80 \\
\hline \hline
\end{tabular}

Nd-YAG laser $(\lambda=1.064 \mu \mathrm{m}, 500 \mathrm{~mW}$ maximum output power, Innolight Mephisto). To measure the free motion of particles, we employed the blinking optical tweezers technique $[25,26]$. This technique allows us to track a single particle in the same focal position for an extended amount of time and for a large number of repeated measurements without moving the sample cell, thus reducing experimental errors due to size distribution or to hydrodynamic interactions among particles in dense samples. The laser beam was switched on and off using a homemade electronic shutter driven by an analog wave form generator and positioned at the focus of the magnifying telescope. The shutter ensures a switching time well below the acquisition time of the CCD camera (i.e., $1 \mathrm{~ms}$ ). The modulation frequency varied between $0.1 \mathrm{~Hz}$ up to a few $\mathrm{Hz}$ depending on the size of the particle used. Videos of the particle motion were acquired using a fast CCD camera with a rate up to 500 fps [see Figs. 2(b) and 2(c)]. We varied the relevant parameters using four types of particle material-polystyrene, melamine, silica, and Licristar (Merck) - and three different fluids - water, acetone, and methanol. Different combinations of these materials allowed us to study the inertial effects under a range of densities and viscosities. The particles were highly diluted to have about a few tens of them in the sample cell, i.e., 5-10 particles/microliter. The relevant properties of the particles and fluids used in this work are reported in Tables I and II, respectively.

\section{RESULTS AND DISCUSSION}

To demonstrate the polynomial increase of the error on the measurements of the MSD, we estimated it from a large number of recorded trajectories of a freely diffusing particle. Figure 3(a) shows the difference of the MSD calculated using the Stokes-Einstein relation with that obtained from experimental values for different particle sizes in different fluids, and Fig. 3(b) shows the corresponding errors in the estimation of the value of $D$. The main contribution to the error in the experiment is due to the fluid viscosity and the limited temporal resolution: This can be understood looking at the relationship for the relaxation time of the fluid $\tau_{\mathrm{f}}$ that becomes longer, i.e.,

TABLE II. Properties of the fluids used in the experiments $\left(T=25^{\circ} \mathrm{C}\right)$.

\begin{tabular}{lcc}
\hline \hline Fluid & $\rho\left(\mathrm{g} / \mathrm{cm}^{3}\right)$ & $\eta(\mathrm{mPa} \mathrm{s})$ \\
\hline Water & 1.00 & 0.89 \\
Methanol & 0.79 & 0.58 \\
Acetone & 0.79 & 0.32 \\
\hline \hline
\end{tabular}
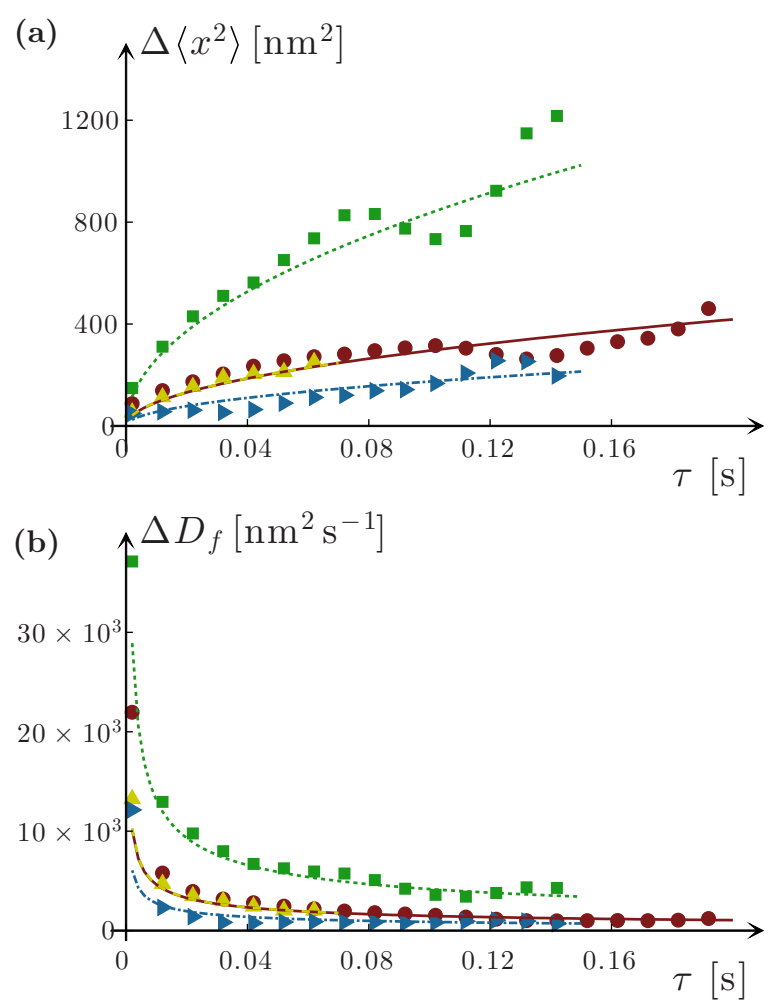

FIG. 3. (Color online) Errors in the determination of the $\Delta\left\langle x^{2}\right\rangle(\tau)$ (a) and of the diffusion coefficient $D$ (b) for different combinations of particles and fluids: $3.1 \mu \mathrm{m}$ Licristar bead in acetone (green squares and dotted line), $5.0 \mu \mathrm{m}$ polystyrene bead in methanol (brown circles and solid line), $4.8 \mu \mathrm{m}$ silica bead in methanol (yellow up triangles and dashed line), and 3.1 $\mu \mathrm{m}$ Licristar bead in water (blue right triangles and dashed-dotted line). The experimental errors are similar to those reported in Fig. 4 and are not shown for clarity.

the error becomes bigger, for either bigger particles radii $R$ or lower fluid viscosities $\eta$. Our results confirm that going from water to methanol to acetone, the error on the MSD increases. This is due to the fact that in lower viscosity fluids, the particles diffuse much faster than in higher viscosity fluids and the effect of the fluid inertia, therefore, lasts longer. It is interesting to note that the effect is clearly visible also in water where most of the experiments relying on diffusion are done. The particles' density, instead, is not a crucial parameter. In fact, the MSD deviation of the $5 \mu \mathrm{m}$ polystyrene and silica particles in the same fluid (methanol) lie on the same theoretical curve, even though the density of silica is roughly twice that of polystyrene.

The results presented in Fig. 3 are limited to $150 \mathrm{~ms}$ since for heavy particles the frequency of the blinking optical tweezers needs to be set high enough in order to limit the fall of the particle due to effective gravity to a range where the blinking optical tweezers is still able to retrap it. In order to overcome this limitation and to measure the deviation for times up to a few seconds, we used melamine particles (diameter $8.1 \mu \mathrm{m})$ in acetone. Due to their weight these particles immediately reach the bottom coverslip, where they perform a quasi-two-dimensional random motion. The particles are prevented from sticking because of the presence of electrostatic 

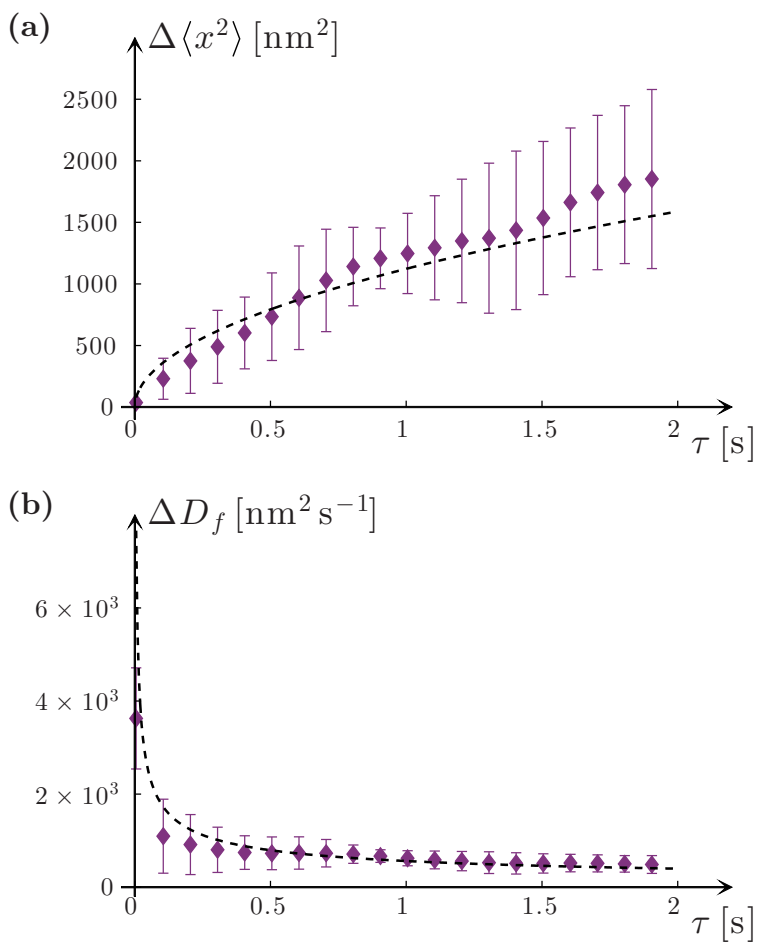

FIG. 4. (Color online) The polynomial behavior of the error in the estimation of (a) the $\Delta\left\langle x^{2}\right\rangle(\tau)$ and (b) of the diffusion coefficient $D$ for an $8.1 \mu \mathrm{m}$ melamine particle up to a few seconds. The fitting to the theoretical model (dashed lines) is used as a guide for the eyes. In fact, the presence of a boundary introduces a change in the polynomial order of the deviation at short time scales that was not included in the model.

repulsive forces. It is worth noting that, since we recorded the motion of the bead in the plane parallel to the surface the anisotropic memory [21] effect does not affect our results. Finally, we used the blinking optical tweezers technique to place the particle at a given initial position and digital video microscopy to record its trajectory. The blinking frequency was set to $0.125 \mathrm{~Hz}$. For each particle, we recorded about 5000 trajectories at $200 \mathrm{fps}$ and calculated the corresponding MSD for each trajectory and then the average. We then averaged the results obtained with five different particles. The resulting deviation between the experimentally measured MSD and the one corresponding to the free diffusion of a massless particle, corrected to take into account the hydrodynamic interaction with the wall [27], is presented in Fig. 4. Although a change in the polynomial order of this deviation is expected at short time scales because of the presence of a boundary $[21,28]$, these experimental data show that the polynomial increase of the error is still an appreciable effect up to a few seconds.

\section{CONCLUSION}

In conclusion, we have demonstrated experimentally that a Brownian particle is subject to inertial effects at long time scales, extending the range of previous experiments by several orders of magnitude up to a few seconds. The measured MSD of a freely diffusing Brownian particle in a liquid shows a deviation from the Einstein-Smoluchowsky theory that diverges with time. These results are consistent with a generalized theory that takes into account the displacement of the fluid surrounding the particle. This can lead to a bias in the estimation of the diffusion from finite-time measurements, as the decay of the relative error is polynomial and not exponential.

\section{ACKNOWLEDGMENTS}

This work was partially supported by the MPNS COST Action 1205 "Advances in Optofluidics: Integration of Optical Control and Photonics with Microfluidics." Giovanni Volpe was partially supported by Marie Curie Career Integration Grant (MC-CIG), Grant No. PCIG11 GA-2012-321726.
[1] A. Rohrbach, C. Tischer, D. Neumayer, E.-L. Florin, and E. H. K. Stelzer, Rev. Sci. Instrum. 75, 2197 (2004).

[2] G. Pesce, G. Volpe, A. C. De Luca, G. Rusciano, and G. Volpe, Europhys. Lett. 86, 38002 (2009).

[3] G. Volpe, G. Volpe, and D. Petrov, Phys. Rev. E 76, 061118 (2007).

[4] A. Imparato, L. Peliti, G. Pesce, G. Rusciano, and A. Sasso, Phys. Rev. E 76, 050101 (2007).

[5] I. Golding and E. C. Cox, Phys. Rev. Lett. 96, 098102 (2006).

[6] B. Wang, S. M. Anthony, S. C. Bae, and S. Granick, Proc. Natl. Acad. Sci. USA 106, 15160 (2009).

[7] E. Barkai, Y. Garini, and R. Metzler, Phys. Today 65(8), 29 (2012).

[8] A. Einstein, Ann. Phys. (NY) 332, 549 (1905).

[9] P. Langevin, C. R. Acad. Sci. (Paris) 146, 530 (1908).

[10] W. Coffey, Y. P. Kalmykov, and J. T. Waldron, The Langevin Equation: With Applications in Physics, Chemistry and Electrical Engineering, Series in Contemporary Chemical Physics (World Scientific, New York, 1996).
[11] B. Øksendal, Stochastic Differential Equations: An Introduction with Applications (Springer, Heidelberg, 2003).

[12] G. Volpe and G. Volpe, Am. J. Phys. 81, 224 (2013).

[13] E. M. Purcell, Am. J. Phys. 45, 3 (1977).

[14] G. E. Uhlenbeck and L. S. Ornstein, Phys. Rev. 36, 823 (1930).

[15] E. J. Hinch, J. Fluid Mech. 72, 499 (1975).

[16] H. J. H. Clercx and P. P. J. M. Schram, Phys. Rev. A 46, 1942 (1992).

[17] B. Lukic, S. Jeney, Z. Sviben, A. J. Kulik, E.-L. Florin, and L. Forro, Phys. Rev. E 76, 011112 (2007).

[18] T. Franosch and S. Jeney, Phys. Rev. E 79, 031402 (2009).

[19] D. S. Grebenkov and M. Vahabi, Phys. Rev. E 89, 012130 (2014).

[20] B. Lukić, S. Jeney, C. Tischer, A. J. Kulik, L. Forró, and E.-L. Florin, Phys. Rev. Lett. 95, 160601 (2005).

[21] S. Jeney, B. Lukić, J. A. Kraus, T. Franosch, and L. Forró, Phys. Rev. Lett. 100, 240604 (2008).

[22] T. Li, S. Kheifets, D. Medellin, and M. G. Raizen, Science 328, 1673 (2010). 
[23] R. Huang, I. Chavez, K. M. Taute, B. Lukic, S. Jeney, M. G. Raizen, and E.-L. Florin, Nat. Phys. 7, 576 (2011).

[24] T. Franosch, M. Grimm, M. Belushkin, F. M. Mor, G. Foffi, L. Forro, and S. Jeney, Nature (London) 478, 85 (2011).

[25] J. C. Crocker and D. G. Grier, Phys. Rev. Lett. 73, 352 (1994).
[26] G. Pesce, G. Rusciano, and A. Sasso, Opt. Express 18, 2116 (2010).

[27] J. Happel and H. Brenner, Low Reynolds Number Hydrodynamics (Springer, New York, 1983).

[28] A. Jannasch, M. Mahamdeh, and E. Schaffer, Phys. Rev. Lett. 107, 228301 (2011). 